\title{
30 Ethische Herausforderungen realer Fälle
}

\author{
Fred Salomon
}

Es gibt unterschiedliche Motive, über ethisch begründetes Handeln nachzudenken. Zum einen kann aus einer philosophischen oder religiösen Weltanschauung mit einem bewusst vertretenen Wertekanon die Überlegung erwachsen, welche Konsequenzen die als richtig erachteten Prinzipien für eine konkrete Situation und die darin möglichen Entscheidungsoptionen haben. Diesem deduktiven Weg, bei dem aus anerkannten moralischen Prinzipien konkrete Handlungen abgeleitet werden, steht in der Alltagspraxis der Medizin der häufiger vorkommende induktive Weg gegenüber. Eine konkrete Situation weckt Fragen nach Begründungen für Entscheidungen, nach größeren Zusammenhängen und übergeordneten Regeln oder Werten. Gerade die naturwissenschaftlich und körperfunktionsorientierte Notfallmedizin, in der rasch pragmatische, oft checklistengestützte Entscheidungen getroffen werden, weckt Fragen nach dem Sinn und der Bewertung des Tuns.

Der seit Mitte des 19. Jahrhunderts zu verzeichnende Zuwachs an Wissen und Fähigkeiten in der Medizin verlangt, über die Anwendung oder den Verzicht auf deren Einsatz zu entscheiden. Wir geraten als Menschen in Situationen, auf deren Zustandekommen wir keinen oder nur begrenzten Einfluss haben. In diesen Situationen müssen wir uns im Rahmen unserer Möglichkeiten verhalten. Existenzphilosophisch betrachtet ist unser Sein ein „Sein in Situationen“ (Jaspers 1932). Das gilt auch für die Notfallmedizin. Selbst wenn ich mir das Berufsfeld ausgesucht habe und mich damit solchen Situationen bewusst zuwende, habe ich auf das Entstehen der konkreten Situation keinen Einfluss.

Sobald uns die Möglichkeit zum Eingreifen gegeben ist - sei es zu reanimieren, Medikamente zu geben oder einen Verletzten rasch zu transportieren -, bin ich unaus- 
weichlich gezwungen, zu entscheiden, ob ich es tue oder welche der Alternativen ich wähle. Auch Abwarten („Ich muss erst noch fragen, nachdenken usw. “) ist die Entscheidung, in diesem Moment nicht einzugreifen. Allen in der Notfallmedizin Tätigen ist bewusst, dass es nur Augenblicke später für eine wirksame Hilfe bereits zu spät sein kann. In der Konfrontation mit einer Notfallsituation maße ich mir also keine Entscheidung an, sondern bin existenziell zu ihr gezwungen. In Weiterführung des existenzphilosophischen Ansatzes sind wir in unserer Grundbefindlichkeit Menschen in der Entscheidung!

\section{[ Entscheiden können heißt entscheiden müssen! \\ - Entschieden haben heißt verantworten müssen! \\ v Verantworten müssen erfordert begründen können!}

Die beeindruckenden Fähigkeiten, Krankheiten behandeln und Lebensbedrohungen abwenden zu können, verlangen ein ethisches Gerüst, um sie verantwortlich anzuwenden, wenn man den freien Willen des Menschen nicht völlig negiert. Die Entscheidungen sind nicht immer leicht zu treffen. Alle verfügbaren Alternativen können problematisch sein. Nicht selten gleicht die Situation dem Dilemma in der antiken Tragödie: Wie man sich auch entscheidet, physische und psychische Verletzungen, Belastung, Trauer oder Schuld sind nicht vermeidbar. Daher ist mit der getroffenen Entscheidung ein Fall oft nicht beendet. Nacharbeit, Überdenken der Entscheidungen und Bewusstmachen der eigenen Emotionen und Maßstäbe sind wichtig, um sich das Abgelaufene klar zu machen und für vergleichbare Situationen vorbereitet zu sein.

Ethische Fallbesprechungen können dabei Hilfe bieten. Fallbesprechungen im Sinne einer Ethikberatung (Salomon 2015) sind für die akute Notfallmedizin aus Zeitgründen nahezu ausgeschlossen. Sie können in der Notfallmedizin jedoch zur Aufarbeitung erlebter Situationen sowie zur Schulung dienen (AC Pflege und Ethik 2005). Zehn reale, anonymisierte Fallbeispiele einer umfangreichen Sammlung aus mehreren Jahrzehnten ärztlicher und notärztlicher Berufspraxis sollen ethische Fragen in unterschiedlich komplexen Situationen verdeutlichen und damit zur Diskussion anregen.

Ethische Probleme sind in der Regel mit einer Fülle anderer Dimensionen verflochten, sodass es nötig ist, die verschiedenen Probleme zu differenzieren. Nur so ist es möglich, die Konflikte zwischen den Beteiligten mit den spezifischen Methoden zu bearbeiten (s. Abb. 17).

Die folgenden Fallbeispiele werden entsprechend dieser Dimensionen aufgeschlüsselt, um die ethischen Fragen herauszufinden. Fragen zu den ethischen Konflikten sollen zum Diskurs anregen, Hinweise auf Ausführungen in den theoretischen Beiträgen des Buches können dabei klären helfen. Didaktische und methodische Empfehlungen geben Tipps, wo und wie die Beispiele zur Schulung genutzt werden können. Die Fragen, Hinweise und Tipps sind Anregungen ohne Anspruch, vollständig zu sein.

Die Autorinnen und Autoren wurden gebeten, ihre Ausführungen in den Theoriebeiträgen durch praxisnahe Fallbeispiele zu veranschaulichen. Diese Beispiele sind in einer Tabelle im Anschluss an die zehn ausführlichen Fälle aufgelistet. So soll erleichtert werden, auch diese Beispiele zu nutzen. 


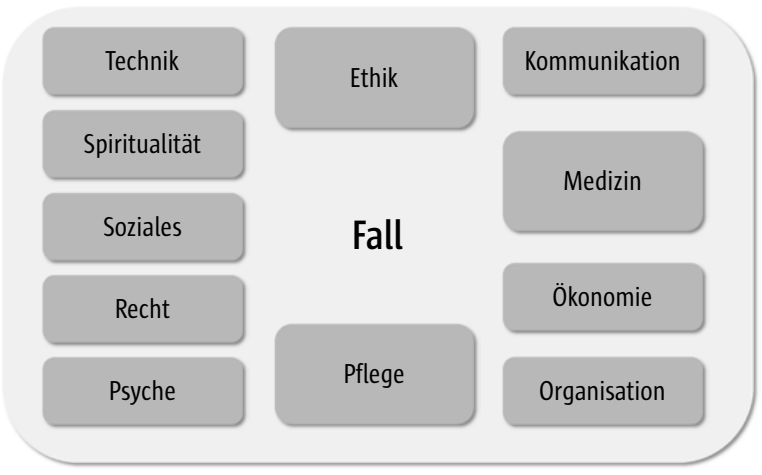

Abb. 17 Die Problemdifferenzierung (alphabetisch geordnet) in einem Fall hilft bei der Analyse der miteinander verflochtenen Dimensionen und ermöglicht es, die jeweiligen Probleme mit den für sie angezeigten Methoden zu bearbeiten.

Alle Fallbeispiele dieses Buches sollen zum Gespräch anregen und einen möglichst herrschaftsfreien Diskurs (Habermas 1983; Kessler 2003) aller Beteiligten fördern. Kommunikation ist zur Klärung ethischer Fragen in der notfallmedizinischen Praxis unverzichtbar. Sie hat damit selbst eine ethische Dimension.

\section{Literatur}

Arbeitsgruppe „Pflege und Ethik“ in der Akademie für Ethik in der Medizin (Hrsg.) (2005) „Für alle Fälle ..." Arbeiten mit Fallgeschichten in der Pflegeethik. Brigitte Kunz Verlag, Hannover

Habermas I (1983) Moralbewusstsein und kommunikatives Handeln. Suhrkamp, Frankfurt/Main Jaspers K (1932) Philosophie. Springer, Berlin

Kessler H (2003) Die philosophische Diskursethik und das Ulmer Modell der Ethikseminare. Ethik Med 15: 258-267 Salomon F (2015) Einrichtung einer Ethikberatung. Med Klinik - Intensivmedizin und Notfallmedizin 110: 204-209

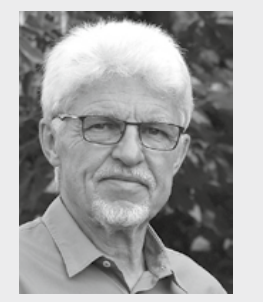

\section{Prof. Dr. med. Fred Salomon}

Studium der Evangelischen Theologie und Medizin. Habilitation für „Ethik in der Medizin“ an der Universität Gießen. Von 1990 bis 2013 Chefarzt der Klinik für Anästhesiologie und Operative Intensivmedizin am Klinikum Lippe-Lemgo. Lehre in „Ethik in der Medizin“ an der Universität Gießen. Ethikberater, Koordinator für Ethikberatung und Trainer für Ethikberatung im Gesundheitswesen (AEM). Vorstandsmitglied der Akademie für Ethik in der Medizin (AEM), Mitglied der AEM-Arbeitsgruppen „Pflege und Ethik“ und „Ethikberatung im Gesundheitswesen“. Mitglied der Sektion Ethik der Deutschen Interdisziplinären Vereinigung für Intensiv- und Notfallmedizin (DIVI). Publikationen zu ethischen Fragen von Intensiv-, Notfall- und Palliativmedizin, Organtransplantation, Menschenbild in der Medizin, Wahrheit am Krankenbett, Grenzfragen zwischen Theologie und Medizin, Ethikberatung und Vermittlung von Ethik. 


\section{Fall 1: Frau Eigen}

\section{Fallskizze}

Frau Eigen, 74 Jahre alt, wohnt allein in einem Bungalow. Es gibt im Ort keine Angehörigen. Es ist Anfang Dezember. Nachbarn fällt auf, dass die Zeitungen von zwei Tagen noch vor der Tür liegen. Als auf Klingeln nicht geöffnet wird, gehen sie ums Haus und sehen durch die Gardine des Wohnzimmers den laufenden Fernseher und die Frau im Sessel sitzend. Da sie auch jetzt nicht reagiert, alarmieren die Nachbarn den Rettungsdienst. Die Leitstelle schickt Feuerwehr und Notarztteam zum Einsatzort, die beide nahezu gleichzeitig eintreffen.

Als die Feuerwehr die Tür öffnet, dringt Fäulnisgeruch aus dem Haus. Aus Nase und Mund der sehr adipösen Frau war bräunliches Sekret gelaufen, das zum Teil schon angetrocknet ist. Die Haut ist livide, ödematös und zeigt an verschiedenen Stellen blasige Ablösungen. Neben ihr steht eine halb gefüllte Tasse mit kaltem Tee. Der Notarzt stellt die Todesursache als ungeklärt fest und benachrichtigt die Polizei. Er füllt nach Untersuchung der Leiche den Leichenschauschein aus, in dem er „Todesursache ungeklärt“" einträgt. Den übergibt er der Polizei, die ihn zunächst bewegen will, bei der alten Frau doch eine natürliche Todesursache zu dokumentieren. Der Notarzt bleibt bei seiner Beurteilung und verlässt mit seinem Team den Einsatzort. Dabei vergisst er, seine Durchschrift des Leichenschauscheins „für Unterzeichner“ mitzunehmen.

Tags darauf ruft er beim Standesamt an, um von dort seine Durchschrift zugeschickt zu bekommen. Man teilt ihm mit, dass von ihm kein Leichenschauschein für die Verstorbene vorliege, sondern ein Leichenschauschein eines niedergelassenen Arztes. Darin sei „Herz-Kreislauf-Versagen“ als Todesursache eingetragen und „natürlicher Tod“" angekreuzt.

\section{Problemdifferenzierung}

ethisch:

Schaden für weitere Fälle vermeiden, Vertrauen in ärztliche Sorgfalt; Vertrauen in der Zusammenarbeit verschiedener Berufsgruppen; Verantwortung der Nachbarn

kommunikativ: Klärung von Meinungsunterschieden zwischen Notarzt und Polizei, Information der Polizei an den Hausarzt

organisatorisch: Regelung der Leichenschau im Rettungsdienst, Rückkoppelung mit Hausarzt bei Unklarheiten

psychologisch: Bestreben der Polizei, den Aufwand bei „Todesursache ungeklärt“ zu vermeiden; Wut des Notarztes über Nichtbeachtung seiner Sorgfalt

juristisch: Leichenschau, Todesursachenklärung, Dokumentation, Ausschluss eventueller Fremdschuld

\section{Ethische Fragen und Problemfelder}

- Welche ethischen Werte begründen die Haltung des Notarztes, die Polizei einzuschalten und die Todesursache staatsanwaltschaftlich klären zu lassen?

- Wie muss oder kann der Notarzt auf den Wunsch der Polizei reagieren, eine natürliche Todesursache zu dokumentieren? 
- Wie ist ethisch und juristisch zu bewerten, dass die Polizei einen weiteren Arzt hinzugezogen hat, der eine „natürliche“ Todesursache dokumentiert?

- Wie hätte der Hausarzt reagieren müssen, wenn er vom Leichenschauschein des Notarztes gewusst hätte?

- Wie sollte der Notarzt auf die Auskunft des Standesamtes reagieren?

\section{Hilfen für Antworten im Theorieteil}

zur Leichenschau: $\quad$ Nüßen

zur Kooperation im Team: Harding, Jaster, Krieter, Marung

\section{Didaktische Hinweise für den Einsatz als Lernbeispiel}

- Schulungsbeispiel für berufsgruppenübergreifende Fortbildungen bei Rettungsdiensten, Polizei, Staatsanwaltschaft und niedergelassenen Ärzten

- Erörterung von Zuständigkeiten bei der Todesfeststellung und den Modalitäten einer Leichenschau

- Erarbeiten möglicher Probleme durch Festlegung als „ungeklärte Todesursache“ einerseits und der Probleme leichtfertiger Feststellung eines „,natürlichen Todes“

- Schulung zur Klärung von Konflikten zwischen Beteiligten 


\section{Fall 2: Kind Ali}

\section{Fallskizze}

Samstag früh um 05:30 Uhr wird das Notarztteam zu Ali, einem 3-jährigen, schwer behinderten Kind, gerufen, weil das Atemüberwachungsgerät die schlafenden Eltern wegen eines Atemstillstands alarmiert hat. Die Eltern sind mit dem potenziell lebensbedrohlichen Krankheitsbild ihres Sohnes gut vertraut, versuchen Ali erfolglos zum Atmen zu bringen und rufen den Rettungsdienst. Die Leitstelle alarmiert das Notarztteam und leitet die Mutter per Telefon zu Reanimationsmaßnahmen an. Das Rettungsteam übernimmt um 05:47 Uhr die Reanimation. Außer der Mutter sind noch der Vater, ein 6-jähriger Bruder, der den Atemstillstand mitbekommen hat, sowie eine Tante des Patienten, die im selben Haus wohnt, anwesend. Tante und Bruder ziehen sich in die Wohnung der Tante zurück. Vater und Mutter assistieren dem Rettungsteam bei der Reanimation des Kindes.

Angesichts der am Telefon erkennbaren Situation hat die Leitstelle parallel zum Notarzt den Notfallseelsorger alarmiert, der um 06:30 Uhr eintrifft. Um 06.35 Uhr erklärt der Notarzt die Reanimation für aussichtslos, während der Monitor eine EKG-Nulllinie zeigt. Das Rettungsteam beendet seine Aktivitäten. Der Vater macht dennoch weiter und wird dabei vom Notarzt angeleitet. Schließlich stellt auch der Vater seine Aktivitäten um 06.40 Uhr ein.

Als der evangelische Notfallseelsorger bemerkt, dass die Familie muslimischen Glaubens ist, bittet er die Leitstelle, einen muslimischen Kollegen zu schicken. Der kann aber frühestens in einer Stunde am Einsatzort sein.

Kurz nach Ende der Reanimation treffen ein mit der Familie befreundetes türkisches Ehepaar sowie vier Vertreter der türkischen Gemeinde ein, die von den Eltern benachrichtigt worden sind. Das befreundete Ehepaar kümmert sich um die Eltern, die Cemeindevertreter um die weitere Versorgung des gestorbenen Kindes. Es herrscht eine große Ruhe. Der Vater dankt als erstes seiner Frau für alles, was sie in den letzten drei Jahren für Ali getan hat, und danach dem Rettungsteam für die Bemühungen, das Leben des Sohnes zu erhalten. Er tröstet sich und alle mit den Worten: „Vielleicht wollte Ali nicht mehr, er wollte nur noch schlafen.“

Um 07:10 Uhr beendet der Notarzt die Leichenschau und füllt die Papiere aus. Danach verlässt das Rettungsteam die Wohnung. Unmittelbar nach Ende der Leichenschau beginnen die männlichen Gemeindevertreter mit dem Vater rituelle Handlungen, waschen Ali, hüllen ihn in eine Decke und tragen ihn in sein Bett im Kinderzimmer.

Tante und Bruder kommen in die Wohnung zurück. Die Mutter entsorgt die letzte Windel und holt Reste von Sondennahrung aus dem Kühlschrank. Danach bedankt sie sich beim Notfallseelsorger, der in der gesamten Zeit ruhig dabei war und vereinzelte kurze Gespräche mit verschiedenen Beteiligten geführt hat. Sie entlässt ihn mit den Worten, dass ihre Familie genügend Unterstützung durch die Freunde und die Gemeinde habe.

\section{Problemdifferenzierung}

ethisch: Achtung der Eltern durch Einbeziehen in Reanimation; Akzep$\operatorname{tanz}$, dass der Vater noch weiter reanimieren will; Akzeptanz von Lebensgrenzen 
kommunikativ: nonverbale Anwesenheit in kritischen Situationen

medizinisch: Therapieversuch; Therapieabbruch; Entscheidung für Beendigung der Reanimation

organisatorisch: Algorithmen an der Leitstelle für Reanimationshilfe per Telefon, Alarmierung von Notarzt, Alarmierung von Notfallseelsorge; Organisation in der türkischen Gemeinde für Beistand in Notsituationen

psychosozial: $\quad$ Umgang mit älterem Bruder; Beistand durch Tante, Notfallseelsorge, Nachbarn, Gemeinde; Gefühl des Notfallseelsorgers angesichts der unterschiedlichen Religionszugehörigkeiten

spirituell: $\quad$ religiöse Bindung der Familie; Trost des Vaters über den „Schlaf“ des Sohnes; Todesriten

technisch: Atemüberwachungsmonitor als Schutz vor Komplikationen; EKGGerät als Hilfsmittel zur Todesfeststellung

\section{Ethische Fragen und Problemfelder}

- Wie ist ethisch und psychisch zu beurteilen, dass die Eltern vom Rettungsteam in die Reanimation ihres Kindes einbezogen werden?

- Wie ist zu beurteilen, dass der Notarzt den Vater bei seinen Reanimationsbemühungen weiter anleitet, obwohl er selbst mit seinem Team die Reanimation bereits als erfolglos beendet hat?

- Wie ist mit der Tante und Alis Bruder umzugehen?

- Wie soll sich der evangelische Notfallseelsorger in der muslimischen Familie verhalten?

- Welche Rolle haben die befreundete Familie und die Vertreter der türkischen Gemeinde für die Familie und welche Konsequenzen ergeben sich daraus für Notfallteam und Notfallseelsorger?

- Cibt es Argumente dafür, die Polizei einzuschalten, z.B. wegen ungeklärter Todesursache?

\section{Hilfen für Antworten im Theorieteil}

\section{zur Reanimation: Boesing}

zum Umgang mit Angehörigen: Boesing, Henze, Lasogga, May, Pompe, Schmidt

zur Notfallseelsorge:

zur Spiritualität:

zur Todesursachenklärung:

zu Emotionen:
Bering, Lammer, Pompe

Boesing, Lammer, Pompe, Schmidt

Nüßen

Henze

\section{Didaktische Hinweise für Einsatz als Lernbeispiel}

- Geeignet, um Mitarbeitende des Rettungsdienstes und Medizinstudierende zu spirituell-religiösen Aspekten eines Notfalls sowie zur Rolle von Angehörigen bei Reanimationen zu schulen

- Schulung von Mitgliedern einer Notfallseelsorge über die unterschiedlichen Riten verschiedener Religionen oder Konfessionen 


\section{Fall 3: Herr Holsten}

\section{Fallskizze}

Herr Holsten, 55 Jahre alt, hat seit knapp zwei Jahren ein Bronchialcarcinom, das nach verschiedenen Therapieansätzen jetzt auch in den Ösophagus eingewachsen ist. Herr Holsten ist verheiratet und hat zwei erwachsene Kinder, die mit ihren eigenen Familien in nächster Nachbarschaft wohnen. Nach dem letzten Krankenhausaufenthalt hat man sich einvernehmlich mit Herrn Holsten, seiner Ehefrau und dem Hausarzt auf eine palliative Therapie verständigt. Es gibt weder eine Patientenverfügung noch eine Vorsorgevollmacht. Herr Holsten ist sich sicher, mit Familie, Hausarzt und Klinik alles besprochen und vereinbart zu haben, und wollte das nicht auch noch schriftlich festlegen. Der Hausarzt kümmert sich um die symptomlindernde Therapie. Ehefrau, Kinder und Schwiegerkinder teilen sich die pflegerischen Aufgaben. Herr Holsten kann mit Hilfe zur Toilette und sitzend auch geduscht werden. Wegen seiner Schwäche liegt er aber die meiste Zeit im Bett oder in einem Liegesessel im Wohnbereich, wo er mit der Familie fernsieht. Er isst nur wenig, trinkt aber ausreichend.

Gegen 22 Uhr bekommt Herr Holsten während des Fernsehens in Anwesenheit seiner Frau und einer Tochter einen Hustenanfall und heftige Atemnot. Die Tochter ruft den Hausarzt an, wird aber über Anrufbeantworter an eine Notrufnummer verwiesen. Über die private Rufnummer, die der Hausarzt der Familie zur Verfügung gestellt hat, ist er auch nicht zu erreichen. Während die Atemnot zunimmt, Herr Holsten heftige Angstsymptome zeigt und schließlich sein Bewusstsein verliert, ruft die Tochter die 112 an.

Das Notarztteam trifft innerhalb weniger Minuten ein und bereitet sofort eine Intubation vor. Auf Hinweis von Ehefrau und Tochter, dass hier keine Versuche zur Lebenserhaltung gemacht werden sollen, reagiert der junge Notarzt mit der Aussage: „Wenn nichts gemacht werden soll, hätten Sie mich nicht rufen dürfen. Ich habe jetzt keine Zeit für Diskussionen. Wir machen, was nötig ist und bringen Ihren Mann in die Klinik. “ Er intubiert Herrn Holsten und bringt ihn beatmet in die Klinik, in der er zuvor mehrfach wegen seines Carcinoms stationär war.

Auf der Intensivstation, wo Herr Holsten bekannt ist, wird das palliative Therapieziel umgesetzt, nachdem Ehefrau und Kinder gegen Mitternacht eingetroffen sind. Die Beatmung wird beendet. Herr Holsten stirbt analgosediert im Beisein seiner Angehörigen.

\section{Problemdifferenzierung}

ethisch:

Autonomie des Kranken; Fürsorge; Therapiezieländerung; Vertrauen von Angehörigen in ärztliche Absprachen; Vertrauen eines Arztes in Angehörigenaussagen

kommunikativ: Absprachen über mögliche Komplikationen bei palliativem Therapieziel; Kommunikation und Sammeln von Informationen unter Zeitdruck im Rettungsdienst

medizinisch: Symptomlinderung bei palliativem Therapieziel; Therapiekonzept für Krisen in einer palliativen Therapie 
organisatorisch: Algorithmen für erwartbare Notfallsituationen festlegen; Therapiebeschlüsse für Dritte nachvollziehbar dokumentieren

ökonomisch: Kosten für eine nicht gewollte und nicht indizierte Therapie psychosozial: Belastung pflegender Angehöriger; Überforderung durch belastende Symptome einer schweren Erkrankung; Konfrontation der Familie mit bevorstehendem Tod

juristisch: $\quad$ Sterben zulassen durch Therapieverzicht

\section{Ethische Fragen und Problemfelder}

- Durch welche Maßnahmen im Vorfeld hätte die invasive Therapie in der Notfallsituation vermieden werden können?

- Ist der Notarzt verpflichtet oder berechtigt, angesichts des Notrufs und der bei Eintreffen erkennbaren Symptome Dyspnoe und Bewusstlosigkeit zu intubieren, zu beatmen und den Patienten in die Klinik zu bringen?

- In welcher psychischen, ethischen und rechtlichen Konfliktsituation befindet sich der Notarzt?

- In welcher psychischen und ethischen Konfliktsituation befinden sich die Angehörigen?

\section{Hilfen für Antworten im Theorieteil}

zur Palliativmedizin:

Luiz, Wiese

zum Patientenwillen:

Luiz, in der Schmitten, Maio, Trzeczak

zum Umgang mit Angehörigen: Pompe, Salomon

zu Emotionen:

Henze, May

zum Handlungszwang: Brosey, Harding, Krieter, Marung, Padberg

zur Ökonomie:

Marckmann

\section{Didaktische Hinweise für den Einsatz als Lernbeispiel}

Geeignet für Schulung von

- Rettungsdienstpersonal aller Berufsgruppen

- Palliativ- und Intensivstationsteams

- Medizinstudierenden

über

- Handlungsautomatismen

- die Notwendigkeit einer klaren Dokumentation von vereinbarten Therapiezielen

- die Beurteilung von Notsituationen durch betroffene Angehörige

- palliative Aspekte im Rettungsdienst 


\section{Fall 4: Herr Palinko}

\section{Fallskizze}

Herr Palinko, 47 Jahre alt, lebt allein in einem alten Gartenhaus. Er ist sehr verwahrlost. Die einzige Bezugsperson ist sein 32-jähriger Neffe, der ihn regelmäßig besucht, ihm Lebensmittel besorgt und sich ein wenig um Ordnung kümmert. Der Neffe findet ihn um 11:oo Uhr nicht ansprechbar, aber ruhig atmend auf dem Boden seiner Wohnung liegend. Er versucht, ihn wiederholt auf die Couch zu setzen. Doch rutscht Herr Palinko immer wieder herunter.

Der Neffe ruft die Hausärztin, die rund 20 Minuten später eintrifft. Es stehen und liegen zwar viele, zum Teil leere Flaschen mit Alkoholika in der Wohnung herum, doch Herr Palinko riecht nicht auffällig nach Alkohol. Während die Hausärztin ihn untersucht, klart er auf und wird renitent. Sie ruft das Ordnungsamt an, von dem ein Vertreter kommt. Da die Voraussetzungen für eine Zwangseinweisung nach dem Gesetz über Hilfen und Schutzmaßnahmen bei psychischen Krankheiten (PsychKG) nicht gegeben sind, rät die Ärztin Herrn Palinko dringend, sich ins Krankenhaus zu begeben. Herr Palinko wird gegen die Ärztin und den Vertreter des Ordnungsamtes ausfallend, während der Neffe die Empfehlung für den Krankenhausaufenthalt unterstützt. Die Ärztin bestellt über die Leitstelle den Krankentransport, stellt eine Einweisung aus und verlässt zusammen mit dem Vertreter des Ordnungsamtes das Haus.

Als der Rettungsdienst ihn abholen will, weigert sich Herr Palinko und wird aggressiv. Auch dem Drängen seines Neffen begegnet er ablehnend: „Du hast mir gar nichts zu sagen!“ Der Rettungsassistent ruft die Hausärztin an und bittet sie, nochmals zu kommen. Die lehnt ab, er solle den Notarzt rufen. Als der Notarzt um 14:oo Uhr eintrifft, lehnt Herr Palinko weiter ab, mit in die Klinik zu kommen. Er will sich einen Kaffee kochen und füllt dabei Tabak in den Filter der Kaffeemaschine. Das Rettungsteam hindert ihn daran, sich daraus ein Getränk aufzubrühen. Es liegen mehrere benutzte Filtertüten mit angetrocknetem Kaffee herum. In einem noch feuchten Filter findet sich auch Tabak, mit dem offenbar vor kurzer Zeit ein Getränk bereitet worden war.

Inzwischen ist ein Freund aus dem Nachbargarten eingetroffen. Der Notarzt weist auf eine mögliche Nikotinintoxikation hin und drängt auf die Mitfahrt in die Klinik. Herr Palinko lehnt vehement ab. Eine kurze Exploration ergibt, dass Herr Palinko zu Person, Zeit, Ort und Versicherungsstatus informiert ist. Nach Aufklärung über mögliche Risiken, die er schimpfend über sich ergehen lässt, unterschreibt er eine Erklärung, dass er gegen dringenden ärztlichen Rat die Mitfahrt verweigert: „Haut endlich ab!" Freund und Neffe wollen bei ihm bleiben. Der Notarzt informiert die Hausärztin und beendet den Einsatz.

\section{Problemdifferenzierung}

ethisch: $\quad$ Konflikt Autonomie - Fürsorge in der akuten Situation und auch in der Dauersituation der Verwahrlosung; Autonomie angesichts Orientierungsstörungen; Vermeiden von Schaden durch Einschalten von Ordnungsbehörden 
kommunikativ: Interaktion bei Aggressivität; Aufklärung über Risiken bei Verdacht auf Intoxikation; Kommunikation mit Neffen und Freund

medizinisch: Klärung einer unklaren Bewusstlosigkeit; Risiko bei Verdachtsdiagnose Nikotinintoxikation; Beurteilung von Entscheidungsfähigkeit

psychologisch: Umgang mit aggressiven, ausfallenden Patienten

juristisch: $\quad$ Kriterien einer Zwangseinweisung; Behandlungspflicht und -ablehnung; Aufgaben von Ordnungsbehörden; Ablehnung einer medizinisch indizierten Maßnahme; Verantwortlichkeit von Notarzt und Hausärztin bei Weigerung durch den Patienten

sozial: $\quad$ Verwahrlosung; Funktion von Bezugspersonen in sozial und gesundheitlich kritischen Situationen

\section{Ethische Fragen und Problemfelder}

- Welche Maßnahmen darf oder muss die Hausärztin ergreifen, um angesichts der offensichtlichen unklaren Bewusstlosigkeit weiteren Schaden für den Patienten zu vermeiden?

- Hätte die Hausärztin beim Patienten bleiben müssen, bis der Rettungsdienst eintrifft?

- Wie ist die Weigerung der Ärztin zu beurteilen, nicht erneut zum Patienten zu kommen, als der Rettungsdienst sie anruft, und stattdessen auf den Notarzt zu verweisen?

- Wie kann der Konflikt zwischen Beachtung der Autonomie und verantwortlicher Fürsorge angesichts der Orientierungsstörung und einer wahrscheinlichen Gefährdung durch die Verwechslung von Kaffee und Tabak gelöst werden?

- Wie ist die Entscheidung des Notarztes zu beurteilen, den Patienten nach Aufklärung und Unterschrift mit den beiden Begleitpersonen in seinem Gartenhaus zu lassen? Was sollte an Verhaltenshinweisen gegeben und eventuell schriftlich fixiert werden?

\section{Hilfen für Antworten im Theorieteil}

zur Einwilligungsfähigkeit: Brosey

zum Patientenwillen:

zur Verantwortung:

zu Emotionen:

zum Recht: in der Schmitten, Maio, Krieter, Trzeczak

Hoffmann, Maio, Padberg, Trzeczak

Henze, Lasogga, May

Brosey, Marung, Nüßen

\section{Didaktische Hinweise für den Einsatz als Lernbeispiel}

Geeignet zur Schulung von Rettungsdienstmitarbeitenden aller Berufsgruppen, Hausärzten, Sozialarbeitern, Ordnungskräften und Medizinstudierenden

v zum Konflikt zwischen Autonomie, Fürsorge und Verantwortung

- dem Umgang mit Aggression, Behandlungsverweigerung und Zwangsmaßnahmen

- Möglichkeiten und Grenzen hausärztlichen Handelns 


\section{Fall 5: Herr Naumann, Frau Zander und Sohn Jörg}

\section{Fallskizze}

Frau Zander bringt ihren 16-jährigen Sohn Jörg morgens um 07:30 Uhr mit dem PKW vom Dorf zur Schule in die nahegelegene Stadt. In einer langgezogenen Linkskurve kommt ihnen Herr Naumann in seinem PKW mit überhöhter Geschwindigkeit entgegen. Er verliert die Gewalt über das Fahrzeug und prallt gegen das Auto von Frau Zander, das gegen einen Straßenbaum geschleudert wird. Das Auto von Herrn Naumann prallt gegen ein Verkehrsschild. Hinter Familie Zander fährt ein LKW, der rechtzeitig stoppen kann und dessen Fahrer unmittelbar danach einen Notruf auslöst, ehe er zu den Unfallfahrzeugen eilt. Von der Leitstelle werden aufgrund der knappen Angaben sofort Polizei, Notarztteam sowie zwei Rettungswagen zum Unfallort geschickt.

Der LKW-Fahrer hilft Frau Zander aus ihrem Auto. Sie blutet an der Stirn, hat Schmerzen im Bereich des Gurtes und kann nur schwer durchatmen. Ihr Sohn ist eingeklemmt, sehr benommen und gibt starke Schmerzen an. In dieser Zeit treffen auch die Polizei und ein Rettungswagen ein. Kurz danach kommen das Notarztteam und der zweite Rettungswagen. Während die Polizei die Unfallstelle sichert, sichten Notärztin und Rettungsteam die drei Verletzten. Frau Zander ist sehr aufgeregt, macht sich Sorgen um ihren Sohn. Sie lässt sich nur widerwillig auf die Trage eines Rettungswagens legen. Herr Naumann ist nicht ansprechbar, atmet aber ausreichend. Er riecht sehr stark nach Alkohol.

Die erfahrene Notärztin lässt Jörg Zander durch eine Rettungsassistentin einen venösen Zugang legen und ein Analgetikum i.v. geben. Sie kümmert sich um Herrn Naumann, der in den zweiten RTW gelegt wird. Er hat abdominelle Prellmarken und zeigt Reaktionen auf lautes Ansprechen. Währenddessen trifft die Feuerwehr ein, die angefordert wurde, um den Eingeklemmten aus dem Wrack zu befreien. Die Ärztin eilt zwischen Herrn Naumann, Frau Zander und Jörg Zander hin und her und erteilt den Rettungsdienstlern Anordnungen. Sie verzichtet darauf, einen zweiten Notarzt oder den Leitenden Notarzt anzufordern, weil die Situation der Verletzten einigermaßen stabil ist, das Krankenhaus in der Nähe liegt und sie ein gutes Team um sich hat. Für Frau Zander wird ein KTW nachgeordert, weil die Ärztin den RTW für Jörg Zander nutzen will, sobald er aus dem Wrack befreit ist.

Aus den Autos, die wegen des Unfalls anhalten mussten, steigen mehrere Personen aus, um näher an das Unfallgeschehen heranzukommen. Einige haben Handys und Kameras in der Hand, um Bilder zu machen. Polizisten versuchen, das zu unterbinden. Eine der Personen weist sich als Journalist der regionalen Zeitung aus und betont sein Recht, Fotos für die Zeitung zu machen.

Frau Zander wird als erste in die Klinik gefahren, obwohl sie ihren Sohn nicht allein lassen will. Als sie in der Notaufnahme eintrifft, die vorher von der Leitstelle über die Art des Unfalls und die zu erwartenden Personen informiert wurde, kritisiert sie heftig den „Blödmann“, der ihnen ins Auto geknallt ist. Mit dem KTW kommen auch Blutentnahmen der beiden anderen Unfallopfer in die Notaufnahme. Kurze Zeit danach treffen Jörg in Begleitung der Rettungsassistentin und Herr Naumann mit der Notärztin ein. Herr Naumann reagiert auf Reize mit Abwehrbewegungen und unverständlichen Lauten. Sein Bauch ist gespannt und deutlich schmerzhaft. 
Ein weiterer Arzt der zur Versorgung in die Notaufnahme kommt, wird vom Oberarzt der Notaufnahme kurz über die drei Verletzten informiert: „Drüben liegt der junge Mann mit Frakturen im Becken-Bein-Bereich, eine davon offen, außerdem gespannter Bauch. In dem andern Raum ist seine Mutter, die vermutlich nur Prellungen und eine Kopfplatzwunde hat. Und hier liegt der Besoffene, 1,9 Promille, der den beiden mit überhöhter Geschwindigkeit ins Auto geknallt ist. Abwehrspannung des Bauches. Für beide Männer Sonografie des Abdomens und CT. Der Junge als Unfallopfer geht vor!“

\section{Problemdifferenzierung}

ethisch: $\quad$ Verantwortung im Straßenverkehr; Datenschutz und Schutz der Intimsphäre der Unfallopfer am Unfallort; Informationsbedarf der Öffentlichkeit und Pressearbeit Gerechtigkeit bei der Verteilung begrenzter Ressourcen in der Notaufnahme; Konflikt durch Vorurteile beim Umgang mit Unfallverursacher und Opfer; Umgang mit vermeintlicher Schuld

kommunikativ: Schlussfolgerungen der Leitstelle aus knappen Informationen bei der Unfallmeldung; Anordnungen der Polizei an Gaffer; Entlastung der Mutter durch Schimpfen; Urteile durch Wortwahl

medizinisch: Sichtung und Prioritätensetzen bei mehreren Verletzten; Analgesie bei Bergung

organisatorisch: Algorithmen bei mehreren Verletzten; Blutproben vom Unfallort vorab in die Klinik schicken

psychologisch: Stress der Mutter durch Sorge um den Sohn; Wut gegen Unfallverursacher; Aggressivität im Behandlungsteam gegen Unfallverursacher und alkoholisiertem Fahrer

juristisch: $\quad$ rechtliche Möglichkeiten der Polizei, gegen Gaffer vorzugehen; Recht der Presse; Rechte der Rettungsassistenten und Verantwortlichkeiten von Notärztin und Rettungsdienstlern

technisch: Hilfsmittel nötig zur Befreiung aus dem Wrack; begrenzte Ressourcen (z.B. 1 CT)

\section{Ethische Fragen und Problemfelder}

- Wie beurteilen Sie die Entscheidung der Notärztin, keinen weiteren Arzt an die Unfallstelle zu holen?

- Welche emotionalen und ethischen Konflikte ergeben sich am Unfallort und in der Notaufnahme daraus, dass der Unfallverursacher alkoholisiert ist?

- Wie beurteilen Sie die Haltung des Oberarztes in der Notaufnahme, das unschuldige Unfallopfer vorrangig zu versorgen?

- Welche Konsequenzen ergeben sich durch die Wortwahl des NotaufnahmeOberarztes bei der Beschreibung des Unfallverursachers für die weiteren Maßnahmen und das Verhalten dem verletzten Mann gegenüber?

- Wie sind ethisch das Fotografieren der Zuschauer und des Journalisten zu differenzieren und zu bewerten? 


\section{Hilfen für Antworten im Theorieteil}

zum Massenanfall von Notfallpatienten: Harding, Reifferscheid zur Schuld:

zur Kommunikation: Fleischmann, Lammer, Pompe zur Öffentlichkeit:

zu Emotionen:

zur Priorisierung: Bering, Marung, May, Padberg Jaster, Krieter, Marung, Parzeller Henze, Hoffmann Harding, Krieter, Marckmann, Marung, Reifferscheid

\section{Didaktische Hinweise für den Einsatz als Lernbeispiel}

Zur Schulung von Rettungsdienstlern aller Berufsgruppen, Notärzten, Verkehrspolizisten, Feuerwehrmitarbeitenden, Mitarbeitenden von Leitstellen und Notaufnahmen, Medizinstudierenden

- zur Verdeutlichung der Probleme bei mehreren Verletzten

- im Umgang mit möglichen Schuldfragen oder Schuldzuweisungen

- beim Umgang mit Gaffern, Angehörigen und Presse

- zum Bewusstmachen der Wirkung von Sprache auf (Vor-)Urteile und Verhalten. 


\section{Fall 6: Herr Demmer}

\section{Fallskizze}

Dirk Demmer, 21 Jahre alt, arbeitslos, wohnt im Haus seiner Eltern. Nach einem heftigen Streit mit seiner Freundin kommt er in den frühen Abendstunden heim. Eine Stunde später verlässt er das Haus, nimmt sich das Auto seiner Eltern und fährt ziellos in der Gegend umher. Auf einem unbeschrankten Bahnübergang wird er von einem Zug erfasst. Der Notarzt findet ihn mit schwerem, offenem Schädel-HirnTrauma bewusstlos neben dem zerstörten Fahrzeug liegen. Intubiert, beatmet und unter Katecholamintherapie kommt Herr Demmer in die Notaufnahme der Uni-Klinik, wo er vom Anästhesie- und Neurochirurgieteam erwartet wird.

Herr Demmer wird sofort ins CT gebracht. Das Schädel-Hirn-Trauma stellt sich als so massiv heraus, dass eine operative Intervention vom Neurochirurgen als aussichtslos angesehen wird. Die Prognose wird von ihm und vom Anästhesisten als „völlig infaust“ bezeichnet. Die weitere Inspektion ergibt nur verschiedene Prellungen und Schürfungen, aber keinen Verdacht auf zusätzliche, behandlungsbedürftige Verletzungen. Herr Demmer zeigt zu diesem Zeitpunkt noch einzelne Atembewegungen und triggert ab und zu das Beatmungsgerät. Der Anästhesist will die Katecholamine abstellen und Herrn Demmer zum Sterben auf die Intensivstation bringen.

Der Neurochirurg widerspricht: „Der junge Mann ist ein hervorragender Organspender. Hier müsst ihr eine organerhaltende Therapie machen!“ Auf den Einwand des Anästhesisten, Herr Demmer sei doch nicht hirntot, er atme schließlich; erwidert sein ärztlicher Kollege: „Das wird aber über kurz oder lang dazu kommen. Wir müssen nur noch etwas abwarten. Organe sind schließlich knapp. “Der Anästhesist hält das für eine nicht vertretbare Instrumentalisierung des Mannes: „Und wenn er dann ein komatöser Pflegefall wird?“ „Der wird bei dem Befund nicht lange überleben“, entgegnet der Neurochirurg.

Die Eltern, die inzwischen auch in der Notaufnahme eingetroffen sind, werden vom Neurochirurgen über den aussichtslosen Befund informiert. Er erwähnt dabei auch vorsichtig die Möglichkeit einer Organentnahme zu Transplantationszwecken. Der Vater legt dem Arzt einen Abschiedsbrief vor, der den Unfall als Suizidversuch erklärt. Außerdem zeigt er ihm eine leere Packung Benzodiazepine, die auf dem Abschiedsbrief im Zimmer des Sohnes lag. Die angesprochene Organspende lehnt die Mutter vehement ab, während der Vater meint, so könne Dirk wenigstens noch etwas Sinnvolles tun.

\section{Problemdifferenzierung}

ethisch: $\quad$ Autonomie und Suizid; Frage des mutmaßlichen Willens des jungen Mannes zur Organspende; Abwägung des Schadens für den jungen Mann bei Warten auf den Hirntod gegen den Schaden für die Menschen, die kein Organ bekommen, wenn der Tod zugelassen wird

kommunikativ: Information der Eltern über die infauste Prognose; Ansprechen von Organspende in der Notaufnahme; Meinungsdissens zwischen den Eltern 
medizinisch: Beurteilung des Befundes nach der Information über einen Suizid mit Verdacht auf Benzodiazepinintoxikation; Therapiezieländerung nach Feststellen der infausten Prognose; eingetretenes oder erwartetes irreversibles Hirnversagen (Hirntod)

organisatorisch: Algorithmen bei möglichen Organspendern; Regeln für den Umgang mit Angehörigen

psychologisch: Belastung der Freundin durch Suizidversuch nach Streit; Belastung des Lokführers durch den schweren Unfall; Belastung des Rettungsteams durch Art des Unfalls; Belastung der Eltern durch Unfall und mitgeteilter Prognose; Beziehung zwischen Mutter und Vater durch unterschiedliche Beurteilung der Organentnahme

juristisch: Voraussetzungen für die Feststellung des irreversiblen Hirnversagens; Einwilligung in Organentnahme nach Transplantationsgesetz

\section{Ethische Fragen und Problemfelder}

- Wie ist ethisch die Meinung des Neurochirurgen zu beurteilen, den jungen Mann weiter zu therapieren, um den noch nicht eingetretenen Hirntod abzuwarten?

n Wie ist ethisch die Position des Anästhesisten zu beurteilen, der die Therapie beenden und den Mann zum Sterben auf die Intensivstation bringen will?

- Wie wäre vorzugehen, wenn der junge Mann einen Organspendeausweis mit Zustimmung zur Organentnahme hätte?

• Wie wäre vorzugehen, wenn der junge Mann eine Patientenverfügung hätte, in der bei festgestellter infauster Prognose auf alle lebenserhaltenden Maßnahmen verzichtet werden und der Tod zugelassen werden soll?

- Was wäre zu beachten, wenn eine derartige Patientenverfügung in Verbindung mit einem Organspendeausweis vorläge?

- Welche Gesichtspunkte ergeben sich medizinisch, ethisch und rechtlich aus dem gefundenen Abschiedsbrief und der leeren Medikamentenschachtel?

- Was bedeutet die unterschiedliche Haltung beider Eltern zur Organspende für die weiteren Maßnahmen und welche ethischen Konsequenzen folgen daraus?

\section{Hilfen für Antworten im Theorieteil}

zum Suizid:

zum Stress:

zum Patientenwillen:

zur Kommunikation:

zum Umgang mit Angehörigen:
Lammer, Lasogga, Pompe

Bering, Marung, May

in der Schmitten, Luiz, Maio, Trzeczak

Bering, Lasogga, May, Padberg

\section{Didaktische Hinweise für den Einsatz als Lernbeispiel}

n Zur Schulung für Mitarbeitende in Notaufnahmen, Ärztinnen und Ärzte, die mit Organspende zu tun haben, Mitglieder von Ethikberatungen, Studierende der Medizin geeignet.

- Thematisch zu nutzen

- für Fragen zum Suizid 
- zur Patientenverfügung

- zum Organspendeausweis

- zum Umgang mit Angehörigen

- Sequenzielle Bearbeitung zu empfehlen: Einschnitt vor Erscheinen der Eltern. 


\section{Fall 7: Stephanie Carl}

\section{Fallskizze}

Die 25-jährige Stephanie Carl hat seit Geburt einen schweren, kombinierten Herzfehler und eine schwerste psychomotorische Retardierung mit multiplen Kontrakturen. Die Mutter ist 64 Jahre alt, der Vater 68. Beide kümmern sich liebevoll um ihr einziges Kind. Stephanie ist intensiv pflegebedürftig. Sie äußert nur verwaschene Laute, die die Eltern zum Teil deuten können, kann nicht selbst essen und trinken, schluckt aber dargereichte Speisen und Getränke langsam. Eine Sonde ist nicht nötig. Tagsüber setzen die Eltern sie in einen speziell für sie angefertigten Stuhl. So ist sie mit am Esstisch, im Wohnzimmer oder auch im Garten.

Sie war mehrfach in verschiedenen Kliniken und ist kardiochirurgisch und orthopädisch operiert worden. Entscheidende Verbesserungen ihrer kardialen und motorischen Situation haben sich dadurch nicht ergeben. Stephanie wird regelmäßig vom Kinderarzt betreut, der sie seit den ersten Lebensjahren kennt. Der Tageslauf der Eltern ist ganz auf den Krankheitszustand ihrer Tochter ausgerichtet. Sie haben nur wenige soziale Kontakte, sind aber in die örtliche Kirchengemeinde eingebunden, deren Angebote die Eltern getrennt nutzen, während der andere bei Stephanie bleibt. Der Pfarrer und einzelne Gemeindemitglieder besuchen die Familie regelmäßig.

Seit den Morgenstunden geht es Stephanie schlechter. Sie frühstückt wenig, atmet schwer und ist unruhiger als sonst. Zwei Stunden später kollabiert sie in ihrem Stuhl und reagiert nicht mehr. Die Eltern legen Stephanie auf den Boden, wie sie es bei früheren Kollapsereignissen taten. Als sich nichts ändert, wählen sie den Notruf 112 und geben neben ihrer Adresse die Information an die Leitstelle: „Unser Kind atmet nicht mehr!“

Das rasch eintreffende Notarztteam stellt einen Atem- und Herzstillstand fest. Während der sofort eingeleiteten Reanimation finden sich eine Nulllinie im EKG und enge Pupillen. Haut und Schleimhäute sind blass-livide. Nach Intubation und ersten Maßnahmen, die zu keiner Herzaktion führen, bitten die Eltern, die Reanimation zu beenden: „Hören Sie auf. Es ist gut so!“ Beide Eltern beschreiben mit knappen, warmen Worten das Leben ihrer Tochter. „Gott hat sie zu sich genommen, jetzt hat Stephanie ihre Ruhe.“ Das Rettungsteam verständigt sich kurz nonverbal, die Reanimation einzustellen.

Der Vater bittet, den Gemeindepfarrer anzurufen. Angesichts der Frage, welche Todesursache im Leichenschauschein nach der sorgfältigen Leichenschau eingetragen werden soll, nimmt der Notarzt auch Kontakt mit dem Kinderarzt auf, der Stephanie gut kennt. Der Pfarrer beschreibt die Familie als sehr verantwortungsbewusst und aufopferungsvoll und sagt zu, im Laufe der nächsten Stunde zu kommen. Er hält jeden Verdacht für unbegründet, dass die Eltern Stephanies Tod herbeigeführt haben könnten. Der Kinderarzt sieht das genauso, deutet den Krankheitszustand als jederzeit potenziell lebensbedrohlich und den Tod damit als natürliche Folge des Herzfehlers. In den Leichenschauschein trägt der Notarzt als natürliche Todesursache „Herzversagen bei bekanntem Herzvitium“ ein. 


\section{Problemdifferenzierung}

ethisch: $\quad$ Fürsorge der Eltern; Entscheidung der Eltern anstelle der nicht entscheidungsfähigen Tochter; Therapiebeendigung angesichts der schwersten Behinderung trotz unzureichender Kenntnis der Anamnese; Vertrauen in Aussage der Eltern; Vertrauen auf Beurteilung durch Pfarrer und Kinderarzt

kommunikativ: nonverbale Abstimmung innerhalb des Rettungsteams über gravierende Entscheidung; Gespräch mit Eltern über Entscheidung des Reanimationsabbruchs; telefonische Kontakte zur Absicherung der notärztlichen Entscheidung

medizinisch: Reanimationsabbruch nach wenigen Minuten bei festgestellter EKG-Nulllinie und engen Pupillen

psychosozial: Belastung der Eltern durch schwerste Behinderung ihrer Tochter seit Geburt

juristisch: Leichenschau im Rettungsdienst; Festlegung der Todesursache; Umgang mit einem nicht auszuschließenden Verdacht auf Tötung der Tochter durch die Eltern

spirituell: $\quad$ Glaubensbindung der Eltern; Einbinden des Cemeindepfarrers

\section{Ethische Fragen und Problemfelder}

- Wie ist zu verstehen, dass die Eltern den Notruf wählen, obwohl sie die Reanimationsmaßnahmen nicht wollen?

- Wie beurteilen Sie die Entscheidung des Notarztes, dem Wunsch der Eltern nach Beendigung der Reanimation sehr früh zu entsprechen?

- Welchen Einfluss kann und darf bei einer solchen Situation die langjährige schwerste Behinderung der Patientin für die Handlungsoptionen in der Notfallsituation haben?

- Wie stehen Sie dazu, dass der Notarzt nach Rücksprache mit Pfarrer und Kinderarzt eine natürliche Todesursache bescheinigt, obwohl er die Patientin, die Anamnese und die aktuelle Notfallsituation kaum beurteilen kann?

- Wäre es gerechtfertigt gewesen, die Polizei einzuschalten und die Todesursache durch die Staatsanwaltschaft klären und ggf. eine Sektion vornehmen zu lassen?

- Was bedeutet für den Fall die kirchliche Bindung und der Glaube der Eltern?

\section{Hilfen für Antworten im Theorieteil}

zur Behinderung:

Bering

zum Umgang mit Angehörigen:

zur Spiritualität:

zur Verantwortung:

Bering, Henze, May

zum Therapieabbruch:

Boesing, Pompe,

Brosey, Jaster

Harding, Luiz, Padberg, Wiese 


\section{Didaktische Hinweise für den Einsatz als Lernbeispiel}

Zur Schulung von Rettungsdienstlern, Notärzten, Notfallseelsorgern, Hausärzten, Kinderärzten und Medizinstudierenden geeignet:

- Behinderung und Notfall

- Anwesenheit von Angehörigen bei Reanimationen

- Therapieentscheidungen durch Eltern

- Leichenschau und Todesursachenfeststellung

- Spiritualität in Notfallsituationen 


\section{Fall 8: Frau Welter}

\section{Fallskizze}

Frau Welter ist 78 Jahre alt. Wegen eines frühkindlichen Hirnschadens mit einer fokal symptomatischen Epilepsie ist sie lebenslang in Betreuung. Seit einigen Jahren wohnt sie in einer Behinderteneinrichtung und braucht sehr viel Hilfe bei den Alltagsaufgaben. Seit mehreren Jahren zeigt sie eine bipolare affektive Störung sowie zunehmende Symptome einer Demenz.

Vor mehreren Jahren wurde die rechte Brust wegen eines Carcinoms entfernt, vor zwei Jahren eine sturzbedingte Oberarmfraktur versorgt. Vor einem Jahr musste sie wegen einer ausgeprägten Anämie durch ein blutendes Ulcus ventriculi bei großer axialer Gleithernie stationär behandelt werden. Die Blutung konnte endoskopisch gestillt werden. Vor sechs Monaten musste eine sturzbedingte Schenkelhalsfraktur operativ versorgt, vor zwei Monaten eine Kopfplatzwunde nach einem Sturz genäht werden.

Das Verständnis für ihre Situation sowie ihre Sturzgefährdung fehlt ihr. Sie ist zeitlich kaum, zu ihrer Person nur begrenzt orientiert, fühlt sich aber in ihrer Wohngruppe in der Behinderteneinrichtung wohl. Ein vom Bereuungsgericht bestellter Berufsbetreuer kümmert sich um anstehende Entscheidungen, hat mit ihr aber nur wenig persönlichen Kontakt.

Anfang Dezember findet ihre Mitbewohnerin, selbst psychisch behindert und betreut, Frau Welter am Boden liegend. Sie möchte ihr helfen sich hinzusetzen. Da das nicht gelingt, ruft sie die diensthabende Pflegerin der Wohngruppe. Die findet Frau Welter bewusstlos auf, stellt eine Bradykardie, eine ausgeprägte Hypotonie sowie eine Schnappatmung fest und alarmiert den Notarzt.

Der Rettungsdienst mit der Notärztin trifft 8 Minuten später ein. Der Zustand Frau Welters veranlasst zur kardiopulmonalen Reanimation mit Herzdruckmassage, Intubation, Beatmung sowie Gabe von Suprarenin und Atropin. Die Mitbewohnerin ist ganz entsetzt und muss aus dem Zimmer begleitet werden. Als innerhalb von $20 \mathrm{Mi}$ nuten keine eigene Herzaktion eintritt und die Pupillen nicht auf Licht reagieren, beschließt die Notärztin den Abbruch der Reanimationsmaßnahmen.

Kurz nachdem alle Tätigkeiten beendet wurden, beginnt das Herz mit einem unregelmäßigen Rhythmus von ca. 6o/min zu schlagen. Einzelne spontane Atemzüge werden danach erkennbar. Die Notärztin nimmt die Therapie wieder auf, gibt kreislaufstabilisierende Medikament und bringt Frau Welter beatmet in die Klinik. Der Blutdruck ist $70 \mathrm{mmHg}$ systolisch. Die Pupillen sind mittelweit ohne Lichtreaktion. Der Cornealreflex ist auslösbar, bei Manipulationen am Tubus aber kein Hustenreflex. Der Bauch ist weich, die Lungen sind beidseits belüftet. Auf der Fahrt zeigt Frau Welter Streckkrämpfe.

In der Notaufnahme ergibt das abdominelle Sonogramm keinen auffälligen Befund. Der $\mathrm{Hb}$ ist 6,o g/dl. Das cerebrale CT zeigt keine Blutung oder Ischämie. Die neurologische Untersuchung ergibt den Verdacht auf einen hypoxischen Hirnschaden. Eine Gastroskopie zeigt viele kleine Schleimhautdefekte mit Zeichen von stattgehabten Blutungen, aber keinen akut therapiebedürftigen Defekt. 


\section{Problemdifferenzierung}

ethisch: $\quad$ Fürsorge für die behinderte Mitbewohnerin; Gutes tun oder Schaden durch Reanimation bei dem schweren, komplexen Krankheitsbild;

kommunikativ: Umgang mit der behinderten Mitbewohnerin; geringer Kontakt zwischen Berufsbetreuer und Patientin; Verständigung im Rettungsteam über Abbruch und Wiederaufnahme der Therapie

medizinisch: Kollaps bei Kombination der Vorerkrankungen; cerebrale Situation bei Abbruch der Reanimation; Indikation zur Wiederaufnahme der Reanimation nach Auftreten von Herzaktionen; Blutgabe bei $\mathrm{Hb}$ von 6,o g/dl und dem neurologischen Befund; Prognose bei Diagnostikende

pflegerisch: Beurteilung der Notfallsituation durch die Pflegende

psychisch: Lebensfreude in der Behinderteneinrichtung; Belastung der behinderten Mitbewohnerin durch den Kollaps und die Notfallmaßnahmen; Belastung der Notärztin und ihres Teams durch spontane Herzaktionen nach Beschluss zur Therapiebeendigung

juristisch: Zuständigkeiten bei Entscheidung über Therapiebegrenzung im Notfall und nach Abschluss der Diagnostik in der Notaufnahme

\section{Ethische Fragen und Problemfelder}

n Wäre es gerechtfertigt, angesichts der komplexen, fortschreitenden Erkrankung auf den Ruf des Notarztes zu verzichten und die Patientin palliativ zu versorgen?

- Wie sollte man mit der behinderten Mitbewohnerin umgehen, die in der Notfallsituation anwesend ist und helfen möchte?

- Wodurch ist der Abbruch der Reanimation nach 20 Minuten gerechtfertigt?

- Wie ist die Wiederaufnahme der abgebrochenen Reanimation nach Eintreten eigener Herzaktionen zu bewerten?

- Welche Handlungsoptionen stellen sich nach Abschluss der Untersuchungen in der Notaufnahme?

- Welche Maßnahmen oder Klärungen im Vorfeld hätten die auftretenden Konflikte in diesem Fall vermindert oder verhindern können?

\section{Hilfen für Antworten im Theorieteil}

zur Reanimation: Luiz, Padberg

zur Behinderung: Bering

zu Therapiezielen: $\quad$ in der Schmitten, Harding, Marung, Padberg, Trzeczak

zum Therapieabbruch: Krieter, Luiz, Trzeczak, Wiese

\section{Didaktische Hinweise für den Einsatz als Lernbeispiel}

- Zur Schulung von Pflegenden in Pflegeinstitutionen, Notärzten, Rettungsdienstmitarbeitenden, Mitarbeitenden in Notaufnahmen und Medizinstudierenden geeignet.

- Zur sequenziellen Bearbeitung geeignet. Dabei Einschnitte nach Abbruch der Reanimation und ggf. auch noch vor Ankunft in der Notaufnahme möglich.

- Schulung zum Umgang mit anwesenden Personen bei Reanimationen und zu Entscheidungen über den Abbruch von Reanimationen. 


\section{Fall 9: Herr Wilkowski}

\section{Fallskizze}

Herr Wilkowski ist 73 Jahre alt und hat vor einigen Jahren einen dreifachen aortokoronaren Bypass sowie einen Aortenklappenersatz bekommen. Wegen plötzlich aufgetretener Rückenschmerzen wird er gegen 19 Uhr in der Klinik seines Wohnortes aufgenommen. Bei der sonografischen und computertomographischen Diagnostik findet sich ein dissezierendes thorakales Aortenaneurysma. Es wird eine Schmerztherapie eingeleitet und eine Verlegung in die ca. $50 \mathrm{~km}$ entfernte Kardiochirurgische Klinik besprochen, in die Sono- und CT-Befunde als Bilddatei geschickt wurden. Herr Wilkowski und seine Frau sind mit Verlegung und eventueller Operation einverstanden. Wegen der weiter bestehenden Schmerzen und einer deutlichen Kreislaufinstabilität wird für die Verlegung das regional verfügbare Intensivmobil angefordert. Es ist schon dunkel, sodass ein Hubschraubertransport nicht schnell verfügbar ist.

Der begleitende Arzt gibt Herrn Wilkowski eine CPAP-Maske mit erhöhter Sauerstoffkonzentration und nimmt eine anxiolytisch-sedierende Medikation vor. Der intraarteriell gemessene Blutdruck ist systolisch zwischen 70 und $80 \mathrm{mmHg}$.

Der Kardiochirurg möchte vor OP noch eine ergänzende Computertomographie des Thorax haben. Der Transport verläuft problemlos. Anästhesieteam und Kardiochirurg nehmen Herrn Wilkowski in der Notaufnahme in Empfang. Während der Kardiochirurg schon in den OP geht, begleitet das Anästhesieteam Herrn Wilkowski zum CT. Beim Umlagern von der Trage auf den Untersuchungstisch bekommt Herr Wilkowski plötzliche Atemnot und wird sehr unruhig. Die arterielle Druckmessung zeigt keine verwertbare Größe an. Ein EKG-Aufkleber fällt ab, sodass kurzzeitig keine technische Information zur Kreislaufsituation besteht.

Als der Anästhesist intubieren will, ist der Kehlkopfeingang durch eine Vorwölbung verschoben und nicht einsehbar. Er ruft: „Tracheotomie“. Bei Verdacht auf eine akute Komplikation der Aortendissektion greift er sofort zum Skalpell und macht einen beherzten Luftröhrenschnitt, durch den der Anästhesiepfleger den Tubus einschiebt. Die Beatmung ist darauf möglich, allerdings blutet es massiv aus der Schnittwunde.

Unter Herzdruckmassage und Beatmung eilen alle sofort in den kardiochirurgischen OP, der vom Radiologen über die Lage informiert wird. Der Patient wird auf der kurzen Fahrt immer blasser. Herzaktionen und Sättigung sind nicht mehr messbar. Die Pupillen sind weit und entrundet. Das OP-Team steht bereit. Als der Tubus am Beatmungsgerät angeschlossen ist, entscheidet der Kardiochirurg: „Keine OP! Wir haben keine Chance mehr. Ich informiere die Klinik, aus der er kommt, und rufe auch seine Angehörigen an.“

Dieser Moment wird als Todeszeitpunkt notiert.

\section{Problemdifferenzierung}

ethisch: Autonomie des Patienten bei Entscheidung zur möglichen OP; Verteilungsgerechtigkeit in einer Gesellschaft durch Verteilung der Versorgungsstrukturen

kommunikativ: Aufklärungsgespräch mit Patient und Ehefrau über mögliche OP und Risiken angesichts der Vorbefunde; rasche Verständigung im 
CT bei akutem Notfall; Aussage im OP zum Verzicht auf OP; Mitteilung des Todes an überweisende Klinik und Angehörige

medizinisch: Indikation zur Verlegung und ggf. OP; Methode der Verlegung und medizinische Versorgung auf dem Transport; CT in aufnehmender Klinik bei schon bekanntem Befund von auswärts; Nottracheotomie; Kriterien zum Abbruch der weiteren Maßnahmen

ökonomisch: regionale Verteilung von speziellen Zentren

organisatorisch: Algorithmen für Notfallverlegungen

psychisch: Belastung des Patienten und seiner Frau durch lebensbedrohliche Diagnose; Belastung der Teams durch plötzlichen Notfall und Tod eines Patienten; Tod auf dem OP-Tisch; Information an Ehefrau über Tod des Mannes

technisch: $\quad$ unzureichende Angaben bei der Herz-Kreislauf-Überwachung

\section{Ethische Fragen und Problemfelder}

- Wie ist der Aufwand für Verlegung und OP bei dem lebensbedrohlichen Aneurysma zu begründen?

n Hätte auf dem Transport bereits eine Sicherung der Atemwege durch Intubation erfolgen müssen?

- Hätte der Kardiochirurg auf ein CT verzichten und sich auf die auswärtigen Bilder verlassen sollen, um Zeit zu gewinnen, als der Patient bei Übernahme in der Notaufnahme noch einigermaßen stabil war?

- Wie ist die Entscheidung des Kardiochirurgen zu bewerten, nicht mehr zu operieren?

- In welcher psychischen Situation befinden sich die verschiedenen Teammitglieder in dieser Situation?

- Wer ist für die Todesfeststellung und wer für die Kommunikation mit den Angehörigen und der aufnehmenden Klinik zuständig?

\section{Hilfen für Antworten im Theorieteil}

zum Patientenwillen: in der Schmitten, Maio, Trzeczak

zur Schuld: $\quad$ Fleischmann, Lammer

zum Therapieabbruch: Luiz, Padberg, Trzeczak

zur Kommunikation: Krieter

zur Teambelastung: Bering, Harding, Hoffmann, Pompe

zur Ökonomie: Marckmann, Salomon

\section{Didaktische Hinweise für den Einsatz als Lernbeispiel}

Geeignet zur Schulung von Ärztinnen, Ärzte, Pflegende, Rettungsdienstmitarbeitende, Mitarbeitende von Notaufnahmen, Medizinstudierende über

- Verhalten in akuten Notfällen

- Entscheidungen zur Therapiebegrenzung und zum Therapieabbruch

- Aufgabenverteilung im Team bei Tod in Notaufnahme und OP 


\section{Fall 10: Frau Sauer}

\section{Fallskizze}

Frau Sauer ist 96 Jahre alt und lebt seit zehn Jahren im Pflegeheim. Drei Kinder und mehrere Enkel kümmern sich regelmäßig um sie. Frau Sauer kann mit dem Rollator noch im Pflegeheim herumgehen, wo sie einige Kontakte pflegt und sich wohl fühlt. Außer ihrer relativen Bewegungseinschränkung hat sie kaum Probleme. Sie ist stark vergesslich und isst und trinkt nur ausreichend, wenn sie immer wieder daran erinnert wird. Im letzten halben Jahr war sie dreimal in der Klinik, weil sie exsikkiert war. Nach einer Infusionstherapie kam sie spätestens zwei Tage später wieder ins Pflegeheim zurück. Der letzte stationäre Aufenthalt war vor einer Woche.

Heute wird sie um 07:0o Uhr kollaptisch in ihrem Bett gefunden. Sie kann sich nicht aufsetzen. Die Hausärztin wird gerufen und bestellt ihrerseits den Notarzt. Bei Eintreffen des Notarztteams zeigt sich eine hypovoläme Frau mit sehr trockener Zunge und stehenden Hautfalten. Der Blutdruck ist $100 \mathrm{mmHg}$ systolisch, die Herzfrequenz 10o/min. Sonst sind keine Auffälligkeiten feststellbar. Frau Sauer reagiert angemessen, wenn auch verzögert auf Ansprechen und ist zur Person orientiert.

Der Notarzt legt einen venösen Zugang und gibt eine Elektrolytlösung. Als die Hausärztin dem Rettungsassistenten den Einweisungsschein für die Klinik geben will, lehnt der Notarzt ab. Er schlägt vor, Frau Sauer im Heim zu lassen, ihr eine gute Flüssigkeitszufuhr zu garantieren und keine invasiven Maßnahmen zu ergreifen, insbesondere keinen erneuten Kliniksaufenthalt zuzumuten.

Die Hausärztin lehnt vehement ab. Sie und das Heim bekämen nur Ärger mit den Angehörigen, wenn Frau Sauer nicht in die Klinik käme. Die Kinder würden auf eine optimale medizinische Versorgung pochen. Die Pflegeleitung der Wohneinheit verweist auch auf den Medizinischen Dienst, der auch immer „Ärger macht“.

Frau Sauer antwortet auf die Frage des Notarztes, was sie den wolle: „Ich weiß auch nicht! Sie sind der Arzt. "Nach einer längeren Diskussion, in der sich die Hausärztin nicht umstimmen lässt, nimmt das Notarztteam Frau Sauer mit in die Geriatrische Abteilung der Klinik.

\section{Problemdifferenzierung}

ethisch: Konflikt von Autonomie und Fürsorge; Verantwortung von Angehörigen, Ärzten, Institution; Konflikt zwischen Gutes tun und Schaden vermeiden

kommunikativ: Gespräch zwischen Hausärztin, Notarzt und Wohngruppenleitung; Gesprächsversuch mit Patientin

medizinisch: Indikation für Klinikaufenthalt; Vermeiden von Hypovolämie bei alten Menschen

ökonomisch: Kostenaufwand für Rettungseinsatz bei Bagatellsituationen; Kosten für Klinikaufenthalt zur Flüssigkeitsgabe

organisatorisch: Garantie einer ausreichenden Ernährung und Flüssigkeitsgabe in Pflegeheimen

pflegerisch: Versorgung trinkunwilliger Bewohner in Pflegeheimen

psychosozial: Beziehung zwischen Kindern und alter Mutter; evtl. Einbinden der Angehörigen in eine pflegerische Versorgung 


\section{Ethische Fragen und Problemfelder}

- Um welche ethischen Werte geht es bei den verschiedenen Handlungsmöglichkeiten der Hausärztin?

- Welche Verantwortung kommt dem Pflegeheim angesichts der wiederholten Volumenmangelereignisse bei der Bewohnerin zu?

- Wie hätte der Notarzt vorgehen können, um der Autonomie der Bewohnerin nach ihrer Aussage „Sie sind der Arzt.“ zu einem größeren Gewicht zu verhelfen?

- Wie sollten die Beteiligten mit dem empfundenen Druck der Angehörigen und des Medizinischen Dienstes umgehen?

- Wie ist ethisch zu werten, dass der Notarzt die Bewohnerin in die Geriatrie mitnimmt?

- Welche Faktoren bei der Patienten (z.B. Alter, wiederholte gleiche Ereignisse) spielen bei dem Entscheidungskonflikt eine Rolle?

- Wie würde die Entscheidung beeinflusst, wenn bei der aktuellen Eksikkose zusätzlich Symptome eines Apoplex vorlägen und die Patientin sich nicht mehr äußern könnte?

- Welchen Einfluss haben ökonomische Erwägungen bei einer solchen Situation?

\section{Hilfen für Antworten im Theorieteil}

zum Alter:

Luiz, Trzeczak, Wiese

zum Patientenwillen: in der Schmitten, Luiz, Maio, Trzeczak

zur Ökonomie: Harding, Marckmann

\section{Didaktische Hinweise für den Einsatz als Lernbeispiel}

Zur Schulung von Pflegenden und Leitenden in Pflegeeinrichtungen, Mitarbeitenden des Medizinischen Dienstes, Hausärzten, Notärzten, Ethikberatungen, Medizinstudierenden geeignet, um institutionelle Rahmenbedingungen, Konflikte zwischen Autonomie und Fürsorge zu erörtern sowie Therapieziele und Pflege zu planen. 


\section{Fallbeispiele in den Theoriebeiträgen}

\begin{tabular}{|c|c|c|}
\hline Autoren & Seite & Fallinhalt \\
\hline Maio & 2 & $\begin{array}{l}\text { Hypoxischer Hirnschaden nach Reanimation. Arzt entscheidet sich zur Therapiereduk- } \\
\text { tion, weil es in solchem Zustand besser wäre zu sterben. }\end{array}$ \\
\hline Maio & 8 & $\begin{array}{l}\text { Querschnittslähmung nach Traktorunfall. Vorhandene Patientenverfügung nach } \\
\text { vorherigem Lymphknotenkrebs. Notwendig werdende Beatmung wird vom Patienten } \\
\text { abgelehnt. Frage nach Ethikkonsil. }\end{array}$ \\
\hline Maio & 12 & $\begin{array}{l}\text { 48-jähriger Mann mit alkoholbedingter Lebercirrhose muss notfallmäßig beatmet } \\
\text { werden. Multiorganversagen. Mutter berichtet vom Todeswunsch des Sohnes. } \\
\text { Diskussion im Team über hohe Kosten der Therapie, schlechte Prognose und Suizidalität. } \\
\text { Bitte um Ethikberatung. }\end{array}$ \\
\hline Harding & 18 & $\begin{array}{l}\text { 46-jährige Frau mit Übelkeit, Erbrechen und Durchfall. Unterschiedliche Beurteilung der } \\
\text { Dringlichkeit und der notärztlichen Einsatzindikation durch Betroffene und Arzt. }\end{array}$ \\
\hline Harding & 23 & $\begin{array}{l}\text { Nicht ansprechbarer 74-jähriger Mann mit akuter Atemnot und Herzinsuffizienz im } \\
\text { Pflegeheim. Keine zutreffende Patientenverfügung, Betreuer nicht erreichbar. }\end{array}$ \\
\hline Krieter & 37 & $\begin{array}{l}\text { Einsatz von Polizei, Feuerwehr, Rettungsdienst mit Notarzt bei er 97-jährigen Frau, die } \\
\text { sich entgegen den Gewohnheiten nicht bei ihrer Tochter gemeldet hat. Große Probleme } \\
\text { nach Aufbrechen der Wohnung mit verwirrt erscheinender und sich wehrender Frau und } \\
\text { der dazu kommenden Tochter. Klärung der Situation, als mit der 97-Jährigen durch das } \\
\text { vorher fehlende Hörgerät eine geordnete Verständigung möglich wird. }\end{array}$ \\
\hline
\end{tabular}

85-jährige Frau kommt aus dem Pflegeheim mit akutem septischem Schock in die

Padberg $49 \quad \begin{aligned} & \text { Notaufnahme. Hindernisse bei Klärung des mutmaßlichen Willens. Entscheidung zur } \\ & \text { Therapiebegrenzung. Trotz zwischenzeitlicher Besserung Therapiezieländerung und } \\ & \text { palliative Versorgung. }\end{aligned}$

Notarzteinsatz bei bewusstseinsgetrübter 81-jähriger Frau in der Kurzzeitpflege nach Hüft-OP. Wegen vital bedrohlicher Situation Mitnahme in die Klinik indiziert. Dann

Luiz 62 Vorlage einer Patientenverfügung mit Ablehnung von Klinikaufnahme und Beatmung. Bei körperlicher Untersuchung Fentanylpflaster entdeckt, die nicht bekannt waren. Nach Naloxongabe klart die Frau auf und stimmt der Mitnahme in die Klinik zu.

\begin{tabular}{|c|c|c|}
\hline Luiz & 65 & $\begin{array}{l}\text { Rettungsdienst alarmiert den Notarzt nach, weil eine 73-jährige, schwerkranke Frau, die } \\
\text { vom Hausarzt in die Klinik eingewiesen wurde, nicht ohne ärztliche Begleitung } \\
\text { transportfähig ist. In Patientenverfügung wird der Tod zuhause gewünscht. Ehemann } \\
\text { schwankt zwischen Wunsch zur Erleichterung für seine Frau und Angst vor Übertherapie. } \\
\text { Notärztlich Bahnung einer palliativen Versorgung in der Klinik. }\end{array}$ \\
\hline Luiz & 66 & $\begin{array}{l}\text { Reanimation in einem Altenheim mit langem Intervall zwischen Notfall und Beginn der } \\
\text { Maßnahmen. Meinungsverschiedenheiten im Rettungsteam über den Abbruch der } \\
\text { Reanimation. }\end{array}$ \\
\hline Luiz & 68 & $\begin{array}{l}\text { Herzinfarkt bei einer 78-jährigen Frau, die ihren pflegebedürftigen Ehemann versorgt } \\
\text { und sich für seine Versorgung trotz ihres akuten Infarktes verantwortlich fühlt. } \\
\text { Einbinden des Ärztlichen Bereitschaftsdienstes zur Versorgung des Ehemannes. }\end{array}$ \\
\hline $\begin{array}{l}\text { in der } \\
\text { Schmitten }\end{array}$ & 85 & $\begin{array}{l}\text { Bei einer 83-jährigen Frau wird ein ausgedehnter, inkurabler Tumor gefunden, der } \\
\text { innerhalb kurzer Zeit zum Tode führen wird. Post-OP Reanimation bei Herzstillstand. } \\
\text { Später OP mit Anus-praeter-Anlage bei lleus. Erst eine Woche danach Verlegung zur } \\
\text { Palliativstation. }\end{array}$ \\
\hline $\begin{array}{l}\text { in der } \\
\text { Schmitten }\end{array}$ & 85 & $\begin{array}{l}\text { 93-jähriger immobiler und schwerst pflegebedürftiger Mann im Pflegeheim. Herzstill- } \\
\text { stand, } 45 \text { Minuten Reanimation ohne Erfolg. }\end{array}$ \\
\hline
\end{tabular}




\begin{tabular}{|c|c|c|}
\hline Autoren & Seite & Fallinhalt \\
\hline $\begin{array}{l}\text { in der } \\
\text { Schmitten }\end{array}$ & 86 & $\begin{array}{l}\text { 78-jährige aktive Frau wird durch einen Schlaganfall schwerst pflegebedürftig. Lange } \\
\text { Therapie ohne Besserung. Erschütterung der Familie über das Ergebnis. }\end{array}$ \\
\hline $\begin{array}{l}\text { in der } \\
\text { Schmitten }\end{array}$ & 88 & $\begin{array}{l}\text { Erfahrener Notarzt empfiehlt als Teilnehmer bei einer Fortbildungsveranstaltung die } \\
\text { Notwendigkeit, auch bei vorliegender Patientenverfügung zu reanimieren, wenn ein } \\
\text { Herzstillstand eintritt. }\end{array}$ \\
\hline $\begin{array}{l}\text { in der } \\
\text { Schmitten }\end{array}$ & 88 & $\begin{array}{l}\text { Frau mit Patientenverfügung und Notfallbogen, in der eine Reanimation abgelehnt wird, } \\
\text { nimmt an einem Hausnotrufsystem teil. Bei Hinweis auf ihre Willensäußerungen wird } \\
\text { ihr mitgeteilt, dass sie trotz ihrer Verfügung in jedem Fall reanimiert würde. }\end{array}$ \\
\hline Trzeczak & 109 & $\begin{array}{l}\text { 67-jähriger Mann mit M. Korsakow als Simulant bei Suizidrisiko stationär eingewiesen. } \\
\text { Übersehener Abszess wird entdeckt und operiert. Morphinmedikation nicht beachtet. } \\
\text { Delir fehlgedeutet. Tod nach Sprung aus dem Fenster. }\end{array}$ \\
\hline Trzeczak & 112 & $\begin{array}{l}\text { 80-jähriger Mann mit kardialer Dekompensation soll aufgrund einer Patientenverfügung } \\
\text { und der Absprache mit der Ehefrau konservativ und palliativ orientiert therapiert } \\
\text { werden. Bei Kammerflimmern auf Drängen der Frau Defibrillation. Meinungsdifferenzen } \\
\text { über Therapieintensität. }\end{array}$ \\
\hline Trzeczak & 116 & $\begin{array}{l}\text { 73-jährige Frau mit Linksherzdekompensation und metastasierendem Tumor. Nach } \\
\text { Überbrücken der Notfallsituation ausführliche Gespräche über Krankheit und Prognose. } \\
\text { Therapieziel palliativ. }\end{array}$ \\
\hline Trzeczak & 116 & $\begin{array}{l}\text { Patient mit komplikationsreichem, langwierigem Verlauf eines Sigmacarcinoms. } \\
\text { Mehrere Operationen und Chemotherapien. Gastrointestinale Blutungen. Nach Gespräch } \\
\text { mit dem Patienten schließlich Verzicht auf weitere invasive Maßnahmen. }\end{array}$ \\
\hline Langer & 147 & $\begin{array}{l}\text { Patientin mit Amnioninfektionssyndrom wird wegen kindlicher Herzaktionen die } \\
\text { Entleerung des Uterus versagt. Sie stirbt daran. }\end{array}$ \\
\hline Lasogga & 171 & $\begin{array}{l}\text { Fall Terri Shiavo: Hypoxischer Hirnschaden. Meinungsdifferenzen über Therapie und } \\
\text { mutmaßlichem Willen zwischen Eltern und Ehemann. Gerichtliche Auseinandersetzung } \\
\text { über Ernährung. Schließlich Tod nach Ernährungsverzicht. }\end{array}$ \\
\hline Wiese & 184 & $\begin{array}{l}\text { Frau mit Larynx-Ca lehnt Klinikeinweisung ab. Notarzt nimmt Kontakt mit Palliativteam } \\
\text { auf und kann die Frau so zuhause lassen. }\end{array}$ \\
\hline Wiese & 184 & $\begin{array}{l}\text { Bei einem Patient mit metastasierendem Prostata-Ca wird eine Reanimation begonnen. } \\
\text { Dabei Klärung des Patientenwillens und der infausten Prognose und Beendigung der } \\
\text { Reanimation. }\end{array}$ \\
\hline Pompe & 202 & $\begin{array}{l}\text { 71-jährige Frau reanimiert unter Anleitung der Leitstelle ihren kollabierten 70-jährigen } \\
\text { Ehemann. Rettungsteam führt die Reanimation ohne Erfolg weiter und bestellt einen } \\
\text { Notfallseelsorger. Notfallseelsorger spricht mit der Frau und dem Sohn, führt rituelle } \\
\text { Handlungen durch und wartet mit den Hinterbliebenen, bis der Bestatter den Toten } \\
\text { abholt. }\end{array}$ \\
\hline Pompe & 204 & $\begin{array}{l}\text { Plötzlicher Kindstod eines drei Monate alten Kindes. Notärztin stellt den Tod fest und } \\
\text { benachrichtigt den Notfallseelsorger. Zur Klärung der Todesursache Einschalten der } \\
\text { Polizei mit Beschlagnahme der Leiche }\end{array}$ \\
\hline Pompe & 207 & $\begin{array}{l}\text { PKW vom Zug erfasst. Belastung der Rettungskräfte durch die Art des Unfalls, die } \\
\text { Unklarheit über die Toten und der Vermutung zu toten Kindern bis zum Eintreffen der } \\
\text { schweren Bergegeräte. Aufgaben für einen Notfallseelsorger. }\end{array}$ \\
\hline Parzeller & 237 & $\begin{array}{l}\text { Verdacht auf Eileiterschwangerschaft in der gynäkologischen Praxis. Die Frau sucht trotz } \\
\text { Risikoaufklärung keine Klinik auf und stirbt nach Eileiterruptur. }\end{array}$ \\
\hline
\end{tabular}




\begin{tabular}{|c|c|c|}
\hline Autoren & Seite & Fallinhalt \\
\hline Marung & 265 & $\begin{array}{l}\text { Im städtischen Gymnasium sind Schusswaffen eingesetzt worden. Die Polizei ist noch } \\
\text { nicht am Ort. Es sind keine Angaben über Verletzte vorhanden. Anordnung an den } \\
\text { Rettungsdienst durch die Leitstelle, zunächst in Distanz zum Einsatzort abzuwarten. }\end{array}$ \\
\hline $\begin{array}{l}\text { Fleisch- } \\
\text { mann }\end{array}$ & $\begin{array}{l}281 / \\
286\end{array}$ & $\begin{array}{l}\text { Assistenzarzt im 1. Weiterbildungsjahr versorgt eine } 36 \text { lahre alte Frau mit einem } \\
\text { Asthmaanfall in der Notaufnahme. Die notwendige Intubation gelingt ihm nicht. Die } \\
\text { Frau stirbt. Probleme im Team und persönliche Belastung des Arztes. Nachfolgende } \\
\text { organisatorische Vereinbarungen zur Verhinderung vergleichbarer Situationen. }\end{array}$ \\
\hline Roshdi & 293 & $\begin{array}{l}\text { Amoklauf Winnenden 2009: 17-lähriger tötet an seiner ehemaligen Schule Schülerinnen, } \\
\text { Schüler und Lehrpersonen. Auf seiner Flucht tötet er auch Unbeteiligte. }\end{array}$ \\
\hline Roshdi & 294 & $\begin{array}{l}\text { Täter am Frankfurter Flughafen tötet } 2011 \text { zwei US-Armeeangehörige und verletzt zwei } \\
\text { schwer. Er sieht sich als Muslim im Krieg mit den USA. }\end{array}$ \\
\hline Roshdi & 295 & $\begin{array}{l}\text { Attentat auf Oskar Lafontaine 1990. Psychotische Frau sticht mit einem Messer in das } \\
\text { Hals des Opfers. }\end{array}$ \\
\hline Roshdi & 296 & $\begin{array}{l}\text { Ein frustrierter Mitarbeiter eines Versandhauses verletzt und tötet mit einem } \\
\text { Samuraischwert mehrere Personen in der Marketingabteilung. Eine Kollegin kann in } \\
\text { stoppen. }\end{array}$ \\
\hline Roshdi & 298 & Patient erschießt nach missglückter Nasen-OP den Chefarzt der HNO-Klinik. \\
\hline Roshdi & 298 & Rentner erschießt zwei Ärzte nach einer Krebsbehandlung. \\
\hline Roshdi & 298 & $\begin{array}{l}1999 \text { erschießt ein 68-jähriger Mann einen Onkologen, dem er die Schuld am Krebstod } \\
\text { seiner Frau gab. }\end{array}$ \\
\hline Lammer & 321 & $\begin{array}{l}\text { Unfallversorgung bei mehreren Verletzten. Ein unbehandelter Patient stirbt. Schuldge- } \\
\text { fühle des Notarztes. }\end{array}$ \\
\hline Lammer & 321 & Notfallseelsorge bei einem Amokläufer, der auf acht Menschen geschossen hat. \\
\hline Lammer & 321 & Umgang mit Schuldgefühlen einer vergewaltigten und verletzten Frau \\
\hline Lammer & 324 & $\begin{array}{l}\text { Schuldgefühle einer Ehefrau, deren Mann stirbt, als sie gerade nicht da ist, obwohl sie } \\
\text { vorher ständig an seinem Bett wachte. }\end{array}$ \\
\hline Lammer & 324 & Selbstvorwürfe eines Mädchens, das von seinem Vater missbraucht wurde. \\
\hline Lammer & 325 & $\begin{array}{l}\text { Schuldgefühle eines Lokführers, der einen Menschen überfahren hat, der ihm vor den } \\
\text { Zug gesprungen ist. }\end{array}$ \\
\hline Lammer & 325 & $\begin{array}{l}\text { Schuldgefühle eines Lehrers, dessen Kollegin bei einem Klassenausflug durch ein Auto } \\
\text { tödlich verletzt wurde. }\end{array}$ \\
\hline Lammer & 325 & LKW-Fahrer, der an einer roten Ampel ungebremst Fußgänger getötet und verletzt hat. \\
\hline Lammer & 326 & Alkoholisierter LKW-Fahrer verursacht einen Unfall mit Toten und Verletzten. \\
\hline Lammer & 326 & Amokläufer hat aus Hass und Rache wahllos auf Menschen geschossen. \\
\hline Lammer & 326 & $\begin{array}{l}\text { Ein Erpresser hat eine Geisel genommen. Er und ein Polizist werden bei einem } \\
\text { Schusswechsel verletzt. }\end{array}$ \\
\hline Henze & 349 & ötzlicher Kindstod. Kümmern um die Mutter. \\
\hline
\end{tabular}




\section{Internetadressen, über die Informationen, Stellungnahmen, Leitlinien und Literatur zu den Themenfragen dieses Buches gefunden werden können (Stand 15.08.2015)}

\section{Akademie für Ethik in der Medizin e.V. (AEM), Göttingen}

Stellungnahmen der Akademie und umfangreiche Links zu Ethik-Institutionen im deutsch- und englischsprachigen Raum, über die man deren Verlautbarungen und Leitlinien finden kann.

Links zu deutsch- und englischsprachigen Zeitschriften zu Ethik in der Medizin.

Link zur Informations- und Dokumentationsstelle Ethik in der Medizin (IDEM), über die man Literaturrecherchen und Expertisen zu medizinethischen Fragen bekommen kann.

http://www.aem-online.de/

\section{Arbeitsgemeinschaft der Wissenschaftlichen Medizinischen Fachgesellschaften (AWMF)}

Arbeitsgemeinschaft einer Vielzahl medizinischer Fachgesellschaften. Auf der Internetplattform finden sich Informationen zu diesen Fachgesellschaften und sehr viele Leitlinien. Darunter sind auch viele ethisch orientierte Stellungnahmen. Es ist eine komfortable Suche nach Fachgesellschafen, Disziplinen, Themen und Relevanz möglich.

www.awmf.org

\section{Bundesärztekammer Berlin}

Stellungnahmen zu vielen ethisch relevanten Themen. Notfallmedizin wird sowohl inhaltlich als auch berufs- und gesellschaftspolitisch thematisiert.

- http://www.bundesaerztekammer.de/

\section{Deutscher Ethikrat, Berlin}

Stellungnahmen des Ethikrates zu ganz unterschiedlichen Themen mit ethischer Relevanz. Links zu nationalen und internationalen Ethikgremien. Speziell zur Ethik in der Notfallmedizin gibt es jedoch wenige Aussagen des Deutschen Ethikrates.

nttp://www.ethikrat.org/

5. Deutsche Gesellschaft für Anästhesiologie und Intensivmedizin (DGAI), Nürnberg Empfehlungen und Vereinbarungen zu verschiedenen Themen auch der Notfallmedizin

http://www.dgai.de/ 


\section{Deutsche Gesellschaft für Internistische Intensivmedizin und Notfallmedizin (DGIIN), Berlin}

Informationen zu Intensiv- und Notfallmedizin. Stellungnahmen und Leitlinien auch $\mathrm{zu}$ verschiedenen ethischen Fragen. Links zu anderen Gesellschaften und Stellungnahmen.

$$
\text { - http://www.dgiin.de/ }
$$

\section{Deutsche Gesellschaft interdisziplinäre Notfall- und Akutmedizin e.V. (DGINA), Berlin}

Interprofessionelle, fachübergreifende Seite zu fachlichen und berufs- und gesellschaftspolitischen Fragen der Notfallmedizin. Ethische Arbeitsgruppe mit eigenen Veröffentlichungen. Blog zur Diskussion.

- http://www.dgina.de/

8. Deutsche Interdisziplinäre Vereinigung für Intensiv- und Notfallmedizin (DIVI), Berlin Informationen und Stellungnahmen zu notfallmedizinischen Fragen. Links zu den Fachgesellschaften in der DIVI.

- www.online-divi.de

\section{9. Österreichische Gesellschaft für Ethik und Recht in der Notfall- und Katastrophenmedizin (öGERN)}

Stellungnahmen, wissenschaftliche Ausarbeitungen und Tagungsberichte zu ethischen und rechtlichen Aspekten für alle Berufsgruppen in der Notfallmedizin. Links $\mathrm{zu}$ vielen vorrangig deutschsprachigen Organisationen und zu Literatur.

n http://www.oegern.at/ogern/

\section{Schweizerische Akademie der Medizinischen Wissenschaften (SAMW), Basel}

Stellungnahmen und Tagungshinweise zu sehr vielen medizinethischen Themen. - http://www.samw.ch/de/Ethik/Richtlinien/Aktuell-gueltige-Richtlinien. html

\section{Schweizerische Gesellschaft für Recht in der Notfallmedizin (GeRN), Muri}

Informationen und Beratungsangebote mit rechtlichem Schwerpunkt. Stellungnahmen, Literaturempfehlungen und Links auch mit ethischem Inhalt, z.B. Autonomie, Aufklärung.

$$
\text { - http://www.notfallrecht.ch/ }
$$

\section{Zentrale Ethikkommission bei der Bundesärztekammer, Berlin}

Stellungnahmen der Zentralen Ethikkommission sowie Links zur World Medical Association mit ethiscehn Stellungnahmen. Notfallmedizin kommt dabei kaum vor.

- http://www.zentrale-ethikkommission.de/ 